\title{
Inhalt, Vol. 10, No. 6, 1987
}

\section{Contents}

Impressum 328

Edler, L. und Flechtner, H., Heidelberg

Remission in Phase-II- und Phase-III-Studien: Kriterien

und Voraussetzungen 330

Hinweise für Autoren 339

Doberauer, C; Niederle, N.; Kloke, O.; Kurschel, E. und Schmidt, C. G., Essen

Zur Behandlung des metastasierten Karzinoids von Ileum und Caecum mit rekombinantem

Interferon alpha-2b. . . 340

Heim, M. E., Mannheim; Fritze, D., Darmstadt; Ho, A.D., Heidelberg; Mebes, W., Mannheim; Abel, U., Heidelberg Phase-III-Studie zur Chemotherapie niedrig-maligner Non-Hodgkin-Lymphome: Vergleich einer Vincristin- mit einer Vindestin-Kombinations-Chemotherapie 345 Hermann, M.; Kober, F. und Keminger, K., Wien Die Fernmetastasierung der Struma maligna. Eine retrospektive Studie über 892 Fälle $\quad 350$ Informationen für die Klinik 356

Wolf, M. und Havemann, K., Marburg; Holle, R. und Drings, P., Heidelberg; Hans, K., Oberhausen;

Schroeder, M., Duisburg

Rezidivhäufigkeit und Langzeit-Überleben beim kleinzelligen Bronchialkarzinom 357

Sonderbände 366

Porzsolt, F. und Schreml, W., Vim; Buchelt, L., Göppingen; Meuret, G. und Mende, S., Ravensburg;

Strigl, P., Schwäbisch Hall; Redenbacher, M., Schwäbisch Gmünd; Klumpp, D., Heidenheim; Schmelz, M., Nürtingen; Knöchelmann, R., Filderstadt; Hiemeyer, V., Kempten; Fleischer, K., Geislingen; Kreuser, E.-D.;

Leichtle, R.; Popp, C. und Kloiber, R., Ulm Konzept zur Behandlung metastasierender Mammakarzinome außerhalb von Universitätskliniken: Beschreibung der Methodeund Prüfung der Effizienz 367 Buchbesprechungen 374

Kurzmitteilung Drings, P., Heidelberg; Calcanis, A., Grenzach Furtulon (Doxifluridin) in der Behandlung des nicht- 
kleinzelligen Bronchialkarzinoms

376

Jahres-Inhalt $1987 \quad 379$

Autorenverzeichnis 1987

383

imprint328

Edler, L. und Flechtner, H., Heidelberg

Remission in Phase II and Phase III Clinical Trials:

Criteria and Prerequisites 330

Instructions for Authors 339

Doberauer, C; Niederle, N.; Kloke, O.; Kurschel, E. and Schmidt, C. G., Essen

Treatment of disseminated ileal and coecal carcincoid

tumors with recombinant alfa-interferon $\quad 340$

Heim, M. E., Mannheim; Fritze, D., Darmstadt; Ho, A. D.,

Heidelberg; Mebes, W., Mannheim; Abel, U., Heidelberg

Chemotherapy of Low Malignancy Non-Hodgkin's

Lymphomas: A Phase III Study Comparing a Vincristine

with a Vindesine Combination Chemotherapy 345

Hermann, M.; Kober, F. and Keminger, K., Vienna

Distant Metastases from Thyroid Carcinoma. A Retro

spective Analysis of 892 Cases $\quad 350$

Clinical Information 356

Wolf, M. and Havemann, K., Marburg; Holle, R. and

Drings, P., Heidelberg; Hans, K., Oberhausen;

Schroeder, M., Duisburg

Patterns of Failure and Long-Term Survival in Small Cell

Lung Cancer (SCLC) 357

Special Editions 366

Porzsolt, F. and Schreml, W., Ulm; Buchelt, L.,

Göppingen; Meuret, G, and Mende, S., Ravensburg;

Strigl, P., Schwäbisch Hall; Redenbacher, M., Schwäbisch

Gmünd; Klumpp, D., Heidenheim; Schmelz, M.,

Nürtingen; Knöchelmann, R., Filderstadt; Hiemeyer, V.,

Kempten; Fleischer, K., Geislingen; Kreuser, E.-D.;

Leichtle, R.; Popp, C. and Kloiber, R., Ulm

Strategy for Treatment of Metastatic Breast Cancer out

side University Hospitals: Description of Methodology

and Efficiency 367

Book Reviews 374

Short Communication

Drings, P., Heidelberg; Calcanis, A., Grenzach

Fortulon (Doxifluridine) in the Treatment of Non Small

Cell Lung Cancer 376

Complete Contents 1987

379

Author Index 1987383

Bibliographischer Hinweis: Inhaltsverzeichmsse dieser Zeitschrift erscheinen regelmäßig in current contents ${ }^{\circledR}$ sowie in anderen bibhographischen Diensten. 